\title{
Estimation of conditions of using combined single-track and double-track railways at high- speed traffic
}

\author{
Alexei Dmitrenko ${ }^{1,{ }^{*},}$ Sergei Karasev $^{1}$, Alexandra Kalidova ${ }^{1}$, and Dmitry Sivitsky ${ }^{1}$ \\ ${ }^{1}$ Siberian Transport University, 630049 Novosibirsk, Russia
}

\begin{abstract}
An important condition when arranging high-speed railway transportation with insignificant traffic is saving of capital investments in construction of new lines or the reconstruction the existing ones. Development of methods for multi-criteria estimation of the possibility of using fully single-track blocks on a line with high-speed traffic, as well as single-track blocks with double-track inserts, is a relevant issue. The purpose of the study is to develop the criteria and the method for estimation of the feasibility of using single-track elements on a line with high-speed traffic. Methods of simulation modeling, mathematical statistics, regression analysis were used. A method that includes estimation of the technological conditions of using single-track elements on a line with high-speed traffic instead of double-track blocks was developed. The method can be used for multi-criteria optimization of the configuration of a combined single-track and a double-track line using single-track elements in high-cost areas.
\end{abstract}

\section{Introduction}

One of the most urgent tasks that railway transport faces is the need for a planned increase in traffic speeds, introduction of high-speed transportation in both passenger and freight traffic [1]. Considering high cost of high-speed transport infrastructure, it is urgent to develop scientifically sound solutions related to the use of combined single-track and double-track lines [2].

The subject of the study is to determine the development logic of single-track lines, to study the influence of parameters of blocks and separate points on the capacity and speed of the train traffic. The scientific problem is in the lack of scientifically justified technical and technological solutions for arrangement of high-speed traffic using combined single-track and double-track lines, which requires development of methods for estimating the use of single-track blocks and artificial structures on specialized lines with high-speed traffic as well as mixed traffic lines.

The lines intended for high-speed traffic are designed to be double-track. This is due to a significant passenger flow, which requires high intensity of traffic of high-speed trains [3]. As a rule, such lines are only specialized in high-speed traffic. High cost of

\footnotetext{
${ }^{*}$ Corresponding author: gdsu@rambler.ru
} 
infrastructure of specialized double-track lines pays off due to the significant traffic volume [4]. With that, in a number of countries in Eastern Europe, Central Asia, South America, as well as in Russia, the volume of passenger traffic is insufficient for development of highspeed traffic under such scheme. In these conditions, creation of a high-speed railway system requires finding ways to reduce the amount of required investments $[5,6]$.

One of the possibilities is the use of single-track distances and artificial structures, separating points [7]. Such solutions ensure significant saving on costs. However, there is almost no scientific research in this direction in the countries that occupy the leading positions in the field of high-speed transportation. The reason is the difference in the volume of passenger traffic: with a large passenger flow, development of single-track lines for high-speed traffic is not considered to be a promising technical solution.

The research is based on findings of a number of authors $[1,2,6,7]$ devoted to the use of single-track elements on high-speed railways, which reduces the infrastructure cost. The experience of using fully or partially single-track lines on the railway network shows that in a number of cases they provide significant saving on capital investments with a sufficient level of capacity $[8,9]$. These studies deal with arrangement of train traffic, scheduling, as well as technical solutions for railway construction.

The authors of this article make an attempt to develop scientific solutions aimed at determining the rational layout of the line. The purpose of the study was to develop the criteria and the method for estimation of the feasibility of using single-track elements on a line with high-speed traffic. The novelty of the study is to consider the problem of using single-track elements on railway lines with high-speed traffic in a multi-criteria setting. The criteria are capacity, train speed, capital costs of line development.

\section{Research methods}

General scientific methods of analysis and synthesis, methods of simulation modeling, mathematical statistics methods are used. The use of single-track blocks with increasing traffic volumes and speed may cause lack of line capacity. This creates the task of estimating the design conditions for single-track blocks.

The method of computer simulation was used to estimate the conditions of single-track elements. Unlike traditional methods of analytical calculation of capacity, the method used provides the possibility of variant calculations with arbitrary parameters of train traffic in both directions. IM Barrier-1 software was developed [10]. Passing of trains through a single-track infrastructure element is simulated (block, artificial construction). The model takes into consideration the structure of missed train traffic, the difference in speed, the duration of occupation of the element, the delay of trains on the approaches and the costs associated with the slowdown in passage of trains.

Approaching of trains to the single-path element can be considered as a stream of demands [5], while the intervals between trains and the duration of occupation of approaches and the element itself can vary. The duration of occupation of the element is directly proportional to its length. It is necessary to assess the influence of single-track blocks parameters on the delays of train traffic and the increase in the transit time of trains.

The program can simulate passing of train traffic in the graphical and analytical form with an arbitrary approach of trains in each direction. Package and non-package schedule is modeled with any given inter-train intervals for an arbitrary time of single-track element occupation by a train in each direction. The incoming flow can be specified as a set of trait attributes $\left\{\Pi_{i}\right\}$ as an object with a set of properties that determine the order of its passing through a single-track element. Such attributes can be, for instance, a high-speed train category, weight and length, a given priority of passing. 
The algorithm of the model is based on combining two incoming train flows from different directions into the general flow, either with or without prioritizing of passing of individual trains (Fig. 1). Selection of the direction priority depends on the following factors: the priority of the train itself; the sequence of approach of trains to a single-track element determined by the schedule or the traffic graph; the train approach speed to the one-track element; the intensity of arrival of trains from each direction.

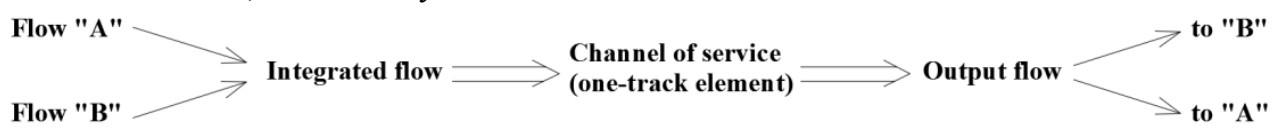

Fig. 1. A single-track element in the form of mass servicing system.

Results of simulation of passing of counter traffic flows are displayed in numerical form. The graph of train passing is also formed, taking into consideration the increase in time on the approaches to the single-track element (Fig. 2).

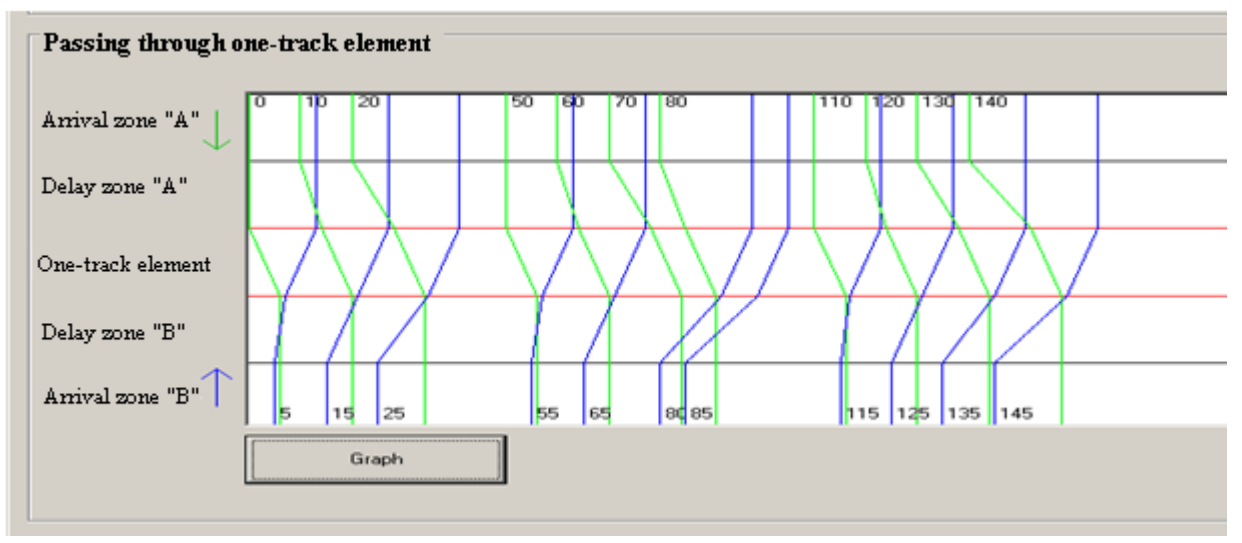

Fig. 2. The graph of passing the counter-moving trains through a one-track block, taking into consideration possible delays due to route hostility.

\section{Results}

The possibility of using single-track or combined single-track and double-track lines is determined by the design and conditions of use of separate points and blocks. One of the obstacles to increase the traffic speed on single-track lines is the design of separate points. The analysis showed that development of increasing volumes of transportation at various stages of development of railway transport took place in the following main ways:

1. By increasing technical equipment of stations, increasing the length of station tracks, taking into consideration the increase in the average weight of freight trains and traffic irregularity, particularly when performing works on the track overhaul.

2. By increasing the capacity of the blocks, first of all, due to construction of doubletrack inserts, and, subsequently, second main tracks.

Passing loops allow increasing the capacity of single-track railway lines. Increase in the number of passing loops reduces the distance between them, reduces the period of the train traffic graph and causes an increase in the line capacity. The nature of passing trains along the main single-track lines is influenced not only by the locations of passing loops, but also by their layout solutions.

In conditions of growth of transportation volumes on single-track railway lines, it is possible to distinguish two basic stages in development of passing loops design. At the first 
stage, in the absence of automated means of communication regarding train traffic, the increase in capacity was ensured by the use of layouts of passing loops and stations with the transverse arrangement of receipt and departure tracks (Fig. 3a). With small initial traffic intensity, the costs associated with stopping of low-weight trains and lengths under crossings at such passing loops are small.
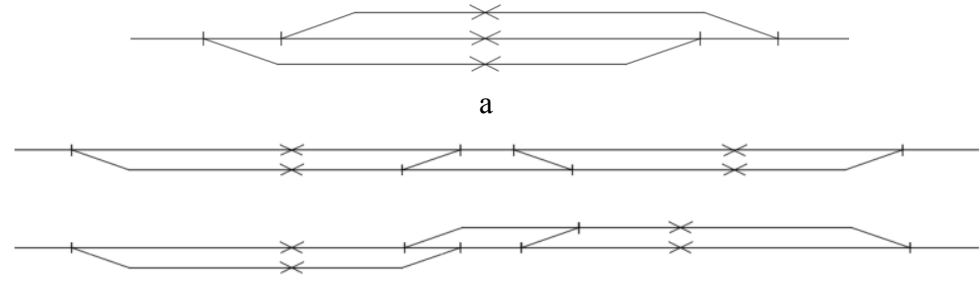

b

Fig. 3. Major passing loops layouts: $a$ - transverse type passing loop; $b$ - longitudinal type passing loop.

At the next stage, development of increasing transportation volumes took place due to the increase in the train traffic speed, the introduction of electric and diesel traction, heavyduty carriages. Non-stop crossing of trains was arranged on single-track railway lines. Development of transportation was also performed through construction of double-track inserts, continuous second main tracks on existing lines. The use of passing loops with the longitudinal arrangement of tracks (Fig. 3b), which provided good opportunities for increasing the speed of passing trains due to non-stop crossing, became more active.

The layout shown in Figure $3 \mathrm{a}$ allows crossing with stopping of freight trains of standard length coming from both even and odd direction. Availability of passing loops with several parallel tracks allows reducing the time spent on preparing tracks for receiving and departure of freight and passenger trains.

It is determined that such passing loops are inefficient in conditions of increasing the length and speed of trains because of the additional costs associated with stopping trains when they cross on single-track lines due to increase in the following:

a) time costs associated with idle trains on the crossing;

b) energy costs associated with braking and acceleration of trains at crossings;

c) total number of crossings due to reduction in traffic speed.

Then, there was an increase in transportation volumes, traffic speed, introduction of new means of communication with trains participating in the traffic. As a result, the efficiency of the use of longitudinal-type passing loops (Fig. 3b) and double-track inserts has increased. Construction of second main tracks became more widespread. However, this measure was not enough to improve the economic performance of single-track railway lines in the face of increasing traffic volumes and the required increase in traffic speed $[11,12]$.

The analysis of the technology of trains passing for layout with transverse arrangement of receiving and departure tracks was performed. The most effective option is when one train stops and the second train passes without stopping (Fig. 4). A completely different situation will arise for highly intensive traffic, when in conditions of crossing irregularity two trains of the opposite traffic direction arrive simultaneously. This will cause the situation when losses of time for parking at crossings will be increased for each of the trains in the layout of passing loops with transverse arrangement of tracks. 


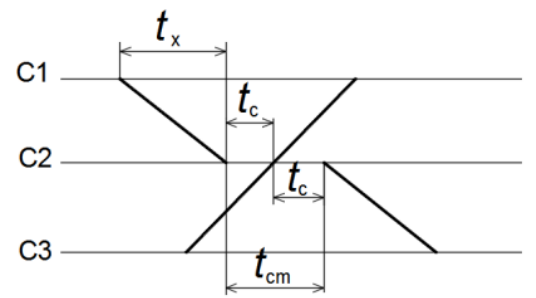

$t_{\mathrm{x}}$ - travelling time; $t_{\mathrm{c}}-$ crossing interval; $t_{\mathrm{cm}}-$ delay at crossing

Fig. 4. Parking layout in the case of simultaneous receiving of trains at their crossing at the station.

Comparison of efficiency of the variants showed that simultaneous receiving at the station is less beneficial for layouts of passing loops with transverse arrangement of tracks. For layouts with longitudinal track arrangement, simultaneous receiving of trains provides a significant reduction in energy costs and the time spent when trains are at the crossing. Besides, if there are transverse-type passing loops, additional tracks are usually not used in the case of subsequent laying of the second main track.

Designing of passing loops with displaced and sequential arrangement of tracks makes it possible to reduce the costs associated with stops. It is of particular importance in the case of simultaneous receiving of trains for crossing.

For the proposed schemes of development of the main single-track railway lines, it is expedient to use long double-track inserts instead of construction of separate passing loop points. They will turn separate blocks or their series into double-track sections (Fig. 5). In this case, there arises the possibility of non-stop crossing of trains running at high speed, as well as long trains. The possibility of trains crossing during package passing will also be improved.

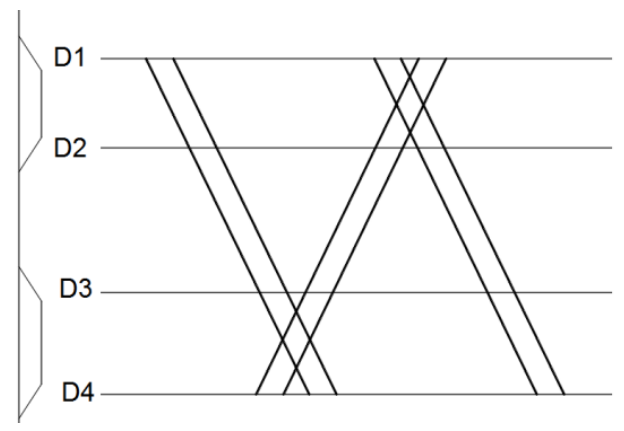

Fig. 5. Train traffic layout in situations of availability of blocks with double-track inserts.

Theoretical analysis of layouts of separate points allows concluding that it is expedient to use passing loops of the longitudinal type in conditions of increasing the traffic speed and development of high-speed traffic. Passing loops with sequential arrangement of tracks on the same side of the main track allows non-stop crossing of trains. It forms good conditions for staged transition to a double-track line. Construction of double-track inserts is recommended at separate stages with a light profile and simple construction conditions. This will allow increasing the train traffic speed with consideration of their crossing without stops. Thus, the subsequent transition from a fully single-track to a combined single-track and double-track line will be performed. Later, it may easily be developed into a double-track line. 
The element that limits the capacity of combined single-track and double-track lines is single-track blocks and other elements (in particular, single-track artificial structures). It is expedient to use simulation methods to determine the possibility of their use in conditions of high-speed traffic. The model allows determining the available capacity of a single-track element (block, artificial structure) for high-speed traffic conditions (Fig. 6).

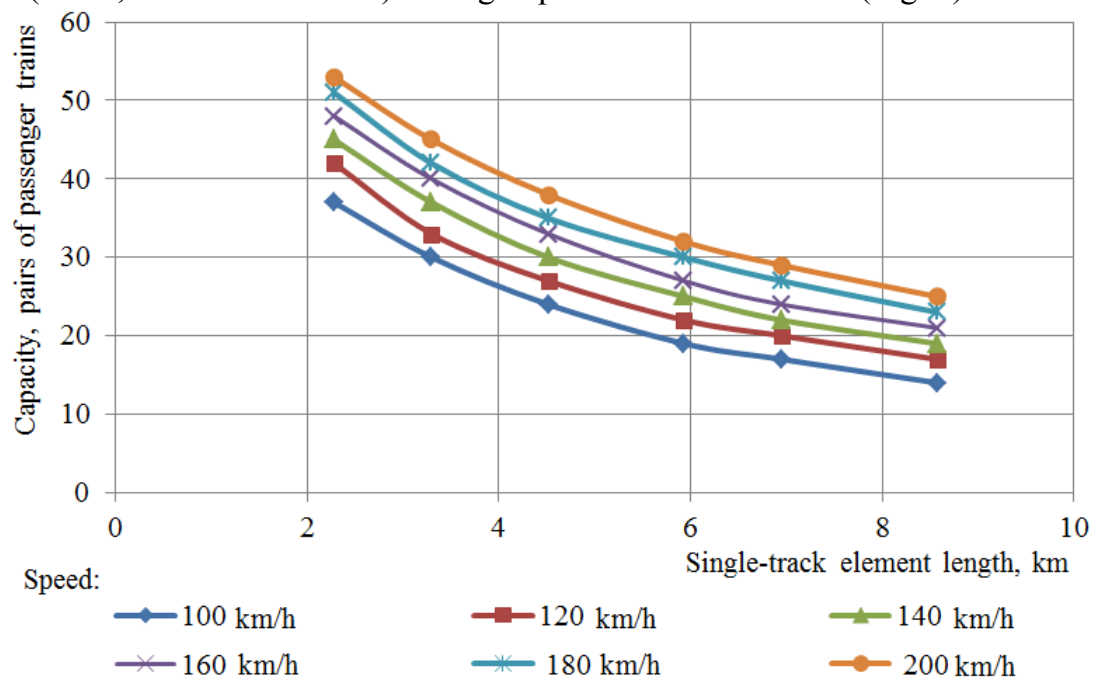

Fig. 6. Graphic dependence of the available capacity on the length of a single-track element.

The dependences obtained can be used to estimate the possibility of using single-track elements on a combined single-track and double-track line. It is also possible to apply them for estimating the capacity level.

The performed theoretical studies and simulation results show that the use of combined single-track and double-track lines can be quite effective at low required capacity. Their application with justified parameters of layouts of separate points and single-track blocks can provide a significant reduction in capital investments at the sufficient level of available capacity.

Arrangement of passing of trains through a single-track element at possible delays depends on the length of the element and the time of its occupation. With consecutive passing of trains, it is necessary to determine the possible delays associated with the route hostility of passing trains. Such delays affect the capacity of a certain element and section. With that, the delay may also be not associated with train stopping. Then, it is necessary to ensure reduction in speed and, correspondingly, increasing in the traveling time on the approach to the single-track element for the period of time equal to the estimated delay time. In this case, the need for a feasibility study for additional development of separate points arises.

In order to ensure the optimal passage of trains, it is necessary to adjust the rate of their approach to single-track blocks. It is achieved by adjusting the intervals between trains. Estimation of conditions of use of single-track elements can be performed by solving the problem of rational distribution of homogeneous resources. Such resources are capital investments or the total travel time of the train over the section. The capacity of line elements in this case is taken into consideration in the form of constraints and can determine the need for conversion of certain single-track elements into double-track ones.

The proposed method makes it possible to reasonably approach making decisions on development of railway networks and individual operating domains, including the 
conditions of increasing traffic speeds. Justified use of combined single-track and doubletrack lines, including the conditions of high-speed traffic will provide a significant reduction in the required investments, make it more realistic to implement similar projects on feeder and other low-loaded lines.

\section{Conclusion}

The results obtained confirm the possibility of using single-track elements on the lines with high-speed train traffic. Single-track elements can provide sufficient capacity for the length of up to several kilometers. The biggest effect in the form of cost savings will be observed on lines with a large number of artificial structures and separating points. The significance of the results obtained lies in the possibility of using them in justifying the infrastructure development of new or reconstructed lines with high-speed traffic. Further research will be devoted to refinement and specification of the results obtained, taking into consideration various means of communication on the train traffic, as well as the forms of the traffic schedule.

\section{References}

1. A.V. Dmitrenko, Vestnik transporta, 10 (2010). (in Russian)

2. E. Castillo, M. Nogal, Z. Grande, Computer-Aided Civil and Infrastructure Engineering, 30 (2015).

3. C Martani, Y Jin, K Soga, S. Scholtes, Computer-Aided Civil and Infrastructure Engineering, 10 (2016).

4. S. Sogin, C.P.L. Barkan, M.R. Saat, Proc. of the Winter Simulation Conference, Vol. 16 (Arizona, 2011)

5. Problems with China's high-speed trains: high costs, safety issues and corruption. Available at: http://factsanddetails.com/china/cat13/sub86/item1848.html (Accessed July 26, 2018).

6. D.Yu. Levin, Mir transporta [World of Transport and Transportation Journal], 2 (2014). (in Russian)

7. S.V. Karasev, A.D. Kalidova, Vestnik of the Railway Research Institute, 1 (2018). (in Russian)

8. X. He, T. Wu, Y. Zou, Y.F. Chen, H. Guo, Structure and Infrastructure Engineering Maintenance, Management, Life-Cycle Design and Performance, Vol. 13 (2017).

9. Sh.-L. Shaw, Zh. Fang, Sh. Lu, R. Tao, Journal of Transport Geography, Vol. 40 (2014).

10. J.E. Doomernik, $13^{\text {th }}$ World Conference on Transportation Research (Rio de Janeiro, Brazil, 2013).

11. M.A. Caldas, F.R. Carvahal, P.D. Gabriele, T.G. Ramos, $13^{\text {th }}$ World Conference on Transportation Research (Rio de Janeiro, Brazil, 2013).

12. M. Nogal, A. O'Connor, B. Caulfield, B. Martinez-Pastor, Rel. Eng. \& Sys. Safety, 156 (2016). 